\title{
Corrigendum: Robust resistive memory devices using solution-processable metal-coordinated azo aromatics
}

Sreetosh Goswami, Adam J. Matula, Santi P. Rath, Svante Hedström, Surajit Saha, Meenakshi Annamalai, Debabrata Sengupta, Abhijeet Patra, Siddhartha Ghosh, Hariom Jani, Soumya Sarkar, Mallikarjuna Rao Motapothula, Christian A. Nijhuis, Jens Martin, Sreebrata Goswami, Victor S. Batista and T. Venkatesan

Nature Materials 16, 1216-1224 (2017); published online 23 October 2017; corrected after print 4 December 2017.

In the version of this Article originally published, the $x$-axis units of Fig. 3 a were incorrectly given as ms, and should have read $\mu$ s. This has now been corrected. Two places in the text also needed amending to reflect this change: the penultimate sentence of Fig. $3 c$,d caption now starts 'Microsecond pulses are used', and the penultimate sentence of the second paragraph of 'Device performance' has been changed to begin 'Device A was measured continuously over 230 days with microsecond write-read pulses'. All have now been corrected in the online versions of the Article.

\section{Corrigendum: Fibronectin-bound $\alpha 5 \beta 1$ integrins sense load and signal to reinforce adhesion in less than a second}

Nico Strohmeyer, Mitasha Bharadwaj, Mercedes Costell, Reinhard Fässler and Daniel J. Müller

Nature Materials 16, 1262-1270 (2017); published online 6 November 2017; corrected online 21 November 2017.

In the version of this Article originally published, Fig. 6 was not cited; a citation to it has now been added at the end of the first paragraph of 'Discussion'. This has been corrected in all versions of the Article. 